International Journal of Difference Equations (IJDE).

ISSN 0973-6069, Volume 16, Number 1, (2021). 169-178

CResearch India Publications

https://dx.doi.org/10.37622/IJDE/16.1.2021.169-178

\title{
Efficiency Sample From Some Sampling Distributions
}

\author{
Mohammed Elmoudier Mohammed Gobar \\ Mathematics Department, Faculty of Science and Arts, \\ Al-Baha University, Kingdom of Saudi Arabia.
}

\begin{abstract}
This paper introduces a method to evaluate the efficiency of a random sample from some sampling distributions. The Shannon entropy and A-entropy are considered to calculate the efficiency of such samples. The obtained results are discussed. An example is given to illustrate the computations of efficiency of random sample from the geometric, binomial and Poisson distributions.

Keywords: Shannon entropy; A-entropy; sup-entropy; efficiency of a random sample; relative efficiency; amount of information.
\end{abstract}

2010 AMS subject classifications: 94A17.

\section{INTRODUCTION}

Sample size determination is an important statistical factor of planning in many fields of studies in statistics. Various criteria may be used to determine sample size [1], [2], [3], [13], [14] and [16]. Measures of information appear in several contexts in probability and statistics, Awad and Al Sarie [5] gave a survey of these measures and some of them are used by author's researches [8], [9], [10], [11] and [12] when the sample size is selected for approximating some sampling distributions by using information measures. This paper aims to derive some results which are analogue to those in [3]. Moreover, the paper suggests a method that evaluates the efficiency of a random sample from different discrete distributions and determine the sample size in order to obtain a sample of a given efficiency. The amount of information in the data is measured by the methods as in [4], [15]. The suggested procedure is tested in cases of geometric, binomial and Poisson distributions. 
Let $\mathrm{X}$ be a random variable in a given probability space $(\Omega, \mathcal{F}, P)$ with probability density function (p.d.f) $f(X ; \theta)$, the Shannon introduced a measure for the amount of information in a random variable $X$ known as "Shannon entropy", which is defined by:

$$
H(X)=-E(\log f(X ; \theta)
$$

where $\mathrm{E}$ denotes for expectation with respect to the probability measure $\mathrm{P}$.

In [4] an extension of the entropy $\mathrm{H}(\mathrm{X})$ defined by Shannon is defined as following:

$$
H(X)=-E\left(\log \frac{f(X ; \theta}{\delta}\right)
$$

where $\delta=\sup _{x} f(x ; \theta)$ which is called A-entropy (known as the sup-entropy).

Definition 1 Let I be an entropy measure and $f(x, y)=f_{1}(x) f_{2}(y)$ where $f(x, y)$ is the joint density of the random vector $(X, Y)$ then

I is said to be additive if $I(f)=I\left(f_{1}\right)+I\left(f_{2}\right)$,

I is said to be subadditive if $I(f) \leq I\left(f_{1}\right)+I\left(f_{2}\right)$

Theorem 1.1 If $S_{n}=\left\{X_{1}, X_{2}, \ldots, X_{n}\right\}$ are independent and identically distributed (i.i.d) random variables of size $n$ from the distribution $f\left(x_{i} ; \theta\right)$, then:

$H\left(S_{n}\right)=n H(X)$ and

$A\left(S_{n}\right)=n A(X)$.

\section{Proof:}

Note that the joint p.d.f of $X_{1}, X_{2}, \ldots, X_{n}$ is

$f\left(x_{1}, x_{2}, \ldots, x_{n}\right)=\prod_{j=1}^{n} f\left(x_{j}\right)$

then

$H\left(S_{n}\right)=-E\left[\log \left(f\left(x_{1}, x_{2}, \ldots, x_{n}\right)\right)\right]=-\sum_{j=1}^{n} E\left[\log \left(f\left(x_{j}\right)\right)\right]=n H(X)$.

Similarly,

$\left.\left(\frac{f\left(x_{1}\right.}{\delta}\right)\left(\frac{f\left(x_{2}\right.}{\delta}\right) \ldots\left(\frac{f\left(x_{n}\right.}{\delta}\right)=\prod_{j=1}^{n} \frac{f\left(x_{j}\right.}{\delta}\right)$

then

$\left.A\left(S_{n}\right)=-E\left[\log \frac{f\left(x_{1}\right.}{\delta}\right)\left(\frac{f\left(x_{2}\right.}{\delta}\right) \ldots\left(\frac{f\left(x_{n}\right.}{\delta}\right)\right]=-\sum_{j=1}^{n} E\left[\log \frac{f\left(x_{j}\right.}{\delta}\right]=n A(X)$.

It is known that the Shannon entropy is additive and subadditive [7].

By additivity of $H\left(S_{n}\right)$ and $A\left(S_{n}\right)$ then the efficiency of such random sample $S_{n}$ is given from it's distributions. To see this let $S_{n}=\left\{X_{1}, \ldots, X_{n}\right\}$ are i.i.d then the Shannon entropy for a random sample $S_{n}$ is given by:

$$
H\left(S_{n}\right)=-\sum_{i=1}^{n} E\left[\log \left(f\left(X_{i}, \theta\right)\right]\right.
$$


and the A-entropy is given by:

$$
A\left(S_{n}\right)=-\sum_{j=1}^{n} E\left[\log \frac{f\left(X_{j}, \theta\right.}{\delta}\right]
$$

where $\delta=\sup _{x_{i}} f\left(x_{i} ; \theta\right)$

Definition 2 Let $X$ be a random variable whose taking values in the set $C:=$ $Y_{1}, Y_{2}, \ldots, Y_{m}$. Assume that we have a random sample $S_{n}$ of size $n$, and let $\left\{x_{1}, x_{2}, \ldots, x_{k}\right\}$ be it's values with frequencies $\left\{r_{1}, r_{2}, \ldots, r_{k}\right\}$, respectively, where $k \leq$ $m$. Now the relative frequency of $x_{i}$ is:

$$
r\left(x_{i}\right)=\frac{r_{i}}{n}, i=1,2, \ldots, k
$$

Hence the estimated Shannon entropy in the sample $S_{n}$ is denoted by

$$
H E\left(S_{n}\right)=-\sum_{i=1}^{k} r\left(x_{i}\right) \log \left(r\left(x_{i}\right)\right)
$$

and the estimated A-entropy in the sample $S_{n}$ is denoted by

$$
A E\left(S_{n}\right)=-\sum_{i=1}^{k} r\left(x_{i}\right) \log \frac{r\left(x_{i}\right)}{\delta}
$$

where $\delta=\max _{x_{i}} r\left(x_{i}\right)$

The Shannon entropy in $X$ is defined by Equation(1.3)and so the estimated relative efficiency in the sample $S_{n}$ is given by:

$$
\text { eff }=\frac{H E\left(S_{n}\right)}{H\left(S_{n}\right)}
$$

where $e f f$ is Shannon relative efficiency.

Also the A-entropy in $X$ is defined by Equation (1.4) and so the estimated relative efficiency in the sample $S_{n}$ is given by:

$$
\text { eff } f^{*}=\frac{A E\left(S_{n}\right)}{A\left(S_{n}\right)} .
$$

where $e f f^{*}$ is Awad relative efficiency. It is known that the amount of entropy in a given sample size is measured by Equations (1.8) and (1.9) are less than or equal to $(1-\beta) 100$ 


\section{MEASURES OF ENTROPY FROM DISTRIBUTIONS}

In this section Shannon and A-entropies are derived for a given discrete distributions.

\subsection{CaseI:Geometric distribution}

Let $Y: G(\theta)$ with p.d.f

$$
f_{1}(y ; \theta)=\theta(1-\theta)^{y-1}, y=1,2, \ldots \text { andzerootherwise }
$$

Let $\delta_{1}=\sup _{y} f_{1}(y ; \theta)$. To calculate Shannon and A-entropies.

Since $\log \left(f_{1}(y ; \theta)\right)=\log \theta+(y-1) \log (1-\theta)$, hence

$$
H_{G}(Y)=-E\left(\log f_{1}(y ; \theta)\right)=-\frac{\theta \log \theta+(1-\theta) \log (1-\theta)}{\theta}
$$

The A-entropy for the $Y: G(\theta)$ from Equation (1.2) is given by:

$$
A_{G}(Y)=H_{G}(Y)+\log \delta_{1}
$$

Let $Y_{1}, Y_{2}, \ldots, Y_{n}$ be a random sample from a Geometric distribution with p.d.f given by Equation (2.1), let $S_{n}=-\sum_{i=1}^{n} Y_{i}$, then by the Theorem1 the Shannon entropy for $S_{n}$ is given by:

$$
H\left(S_{n}\right)=n H_{G}(Y)
$$

and the A-entropy for $S_{n}$ is:

$$
A\left(S_{n}\right)=n A_{G}(Y)
$$

\subsection{CaseII:Binomial distribution}

Let $Y_{1}, Y_{2}, \ldots, Y_{n}$ be a random sample from a Bernoulli distribution with p.d.f $f(y ; \theta)=\theta^{y}(1-\theta)^{n-y}$ if $y \in 0,1$ and zero otherwise.

Let $X=\sum_{i=1}^{n} Y_{i}$; notice that $X: B(n, \theta)$ with p.d.f $f_{2}(x ; \theta)=\frac{n !}{(n-x) ! x !} \theta^{x}(1-\theta)^{n-x}$ if $x \in 0,1,2, \ldots, n$ and zero otherwise Let $\delta_{2}=\sup _{x} f_{2}(x ; \theta)=f(\lceil\theta(n+1)\rceil ; \theta), n \geq 2$ whenever $\lceil k\rceil$ is the greatest integer less than or equal to $k$. then the Shannon entropy is

$$
\begin{aligned}
H_{B}(X) & =-E\left(\log \left(f_{2}(x ; \theta)\right)\right) \\
& =-E(\log (x !(n-x) !))+\log (n !)+n \theta \log \theta+n(1-\theta) \log (1-\theta))
\end{aligned}
$$


Then A-entropy for the $X$ is given by:

$$
A_{B}(X)=H_{B}(X)+\log \delta_{2}
$$

where $\theta^{\wedge}=x$.

\subsection{CaseIII:Poisson distribution}

Let $Y_{1}, Y_{2}, \ldots, Y_{n}$ be a random sample from a Poisson distribution with p.d.f $f(y ; \theta)=\frac{e^{-\theta} \theta^{y}}{y !}$ if $y \in 0,1,2, \ldots$ and zero otherwise.

Let $X=\sum_{i=1}^{n} Y_{i}$, that is for large $n$ and small $\theta, X \approx$ Poisson distribution with $n \theta$ [6].The approximate p.d.f of $X$ is given by

$f_{3}(x ; \theta)=\frac{e^{-n \theta}(n \theta)^{x}}{x !}$ if $x \in 0,1,2, \ldots$ and zero otherwise

notice that:

$\delta_{3}=\sup _{x} f_{3}(x ; \theta)=f_{3}(\lceil n \theta\rceil ; \theta), n \geq 2$

and

$\theta^{\wedge}=\frac{X}{n}$.

from which $\log f_{3}(x ; \theta)=-n \theta+x \log n \theta-\log x !$,

Then the Shannon entropy is:

$$
H_{P}(X)=-E\left(\log \left(f_{3}(x ; \theta)\right)\right)=n \theta(1-\log n \theta)+E(\log x !)
$$

It can be shown that the A-entropy is given by:

$$
A_{P}(X)=E(\log x !)-\log (\lceil n \theta\rceil !)-(n \theta-\lceil n \theta\rceil) \log (n \theta) .
$$

\section{RESULTS}

Throughout this section, the Matlab 8.1 program have been used to perform the computations. The estimate of the Shannon entropy,A-entropy and the efficiency in the sample $S_{n}$ are given by Equations (1.8)and(1.9)in each Case I ,II and III.

\subsection{Example}

a)To illustrate the concepts, as in section 2.1 CaseI, 80 random samples are simulated from geometric distributions: $G(\theta)$. For each $\theta=0.2$ : 0.1 : 0.9 and for each $n=10: 10: 100$, in each case the efficiency ef $f$ and ef $f^{*}$ of each simulated sample is computed in Tables 1 and 2 from Equations (2.4) and (2.5) respectively. 
Table 1: Estimated efficiency of sample of size $n$ from geometric distribution with parameter $\theta$ by Shannon entropy

\begin{tabular}{|c|c|c|c|c|c|c|c|c|}
\hline$n$ & 0.2 & 0.3 & 0.4 & 0.5 & 0.6 & 0.7 & 0.8 & 0.9 \\
\hline 10 & 0.6110 & 0.6158 & 0.6732 & 0.4046 & 0.5957 & 0.6764 & 0.4331 & 0.0000 \\
\hline 20 & 0.6703 & 0.5730 & 0.5969 & 0.7797 & 0.5877 & 0.6764 & 0.4331 & 0.9752 \\
\hline 30 & 0.7083 & 0.6423 & 0.6708 & 0.6934 & 0.8065 & 0.8589 & 0.9533 & 0.6646 \\
\hline 40 & 0.7612 & 0.7449 & 0.6854 & 0.7749 & 0.7933 & 0.7900 & 0.7103 & 0.6146 \\
\hline 50 & 0.6823 & 0.8251 & 0.6899 & 0.9437 & 0.8343 & 0.5672 & 0.7117 & 0.0000 \\
\hline 60 & 0.7584 & 0.7479 & 0.7534 & 0.7286 & 0.9050 & 0.7709 & 0.8947 & 0.6618 \\
\hline 70 & 0.7604 & 0.8045 & 0.7548 & 0.8120 & 0.7004 & 0.6708 & 0.7456 & 0.4082 \\
\hline 80 & 0.7519 & 0.7259 & 0.8199 & 0.7344 & 0.7971 & 0.7880 & 0.6778 & 0.6146 \\
\hline 90 & 0.8401 & 0.8602 & 0.7475 & 0.7341 & 0.8236 & 0.6806 & 0.7567 & 0.9553 \\
\hline 100 & 0.8281 & 0.7898 & 0.8273 & 0.8976 & 0.8586 & 0.8195 & 0.8789 & 0.9652 \\
\hline
\end{tabular}

Table 2: Estimated efficiency of sample of size $n$ from geometric distribution with parameter $\theta$ by A-entropy

\begin{tabular}{|c|c|c|c|c|c|c|c|c|}
\hline$n$ & 0.2 & 0.3 & 0.4 & 0.5 & 0.6 & 0.7 & 0.8 & 0.9 \\
\hline 10 & 0.1637 & 0.3404 & 0.1788 & 0.3805 & 0.2870 & 0.2518 & 0.4064 & 0.6083 \\
\hline 20 & 0.3451 & 0.2565 & 0.2633 & 0.3392 & 0.3564 & 0.4076 & 0.4160 & 0.7203 \\
\hline 30 & 0.2614 & 0.3376 & 0.2346 & 0.4216 & 0.5289 & 0.5710 & 0.5622 & 0.7426 \\
\hline 40 & 0.3321 & 0.4539 & 0.2460 & 0.4651 & 0.5221 & 0.5281 & 0.5486 & 0.5217 \\
\hline 50 & 0.2538 & 0.4862 & 0.5133 & 0.3726 & 0.5486 & 0.5154 & 0.6120 & 0.9295 \\
\hline 60 & 0.3243 & 0.4005 & 0.4819 & 0.4951 & 0.4365 & 0.5907 & 0.6135 & 0.5532 \\
\hline 70 & 0.2706 & 0.3450 & 0.3963 & 0.5608 & 0.6174 & 0.5781 & 0.5744 & 0.8372 \\
\hline 80 & 0.2521 & 0.3328 & 0.3507 & 0.4764 & 0.5317 & 0.6281 & 0.5517 & 0.8795 \\
\hline 90 & 0.3072 & 0.3960 & 0.3936 & 0.4360 & 0.4181 & 0.5409 & 0.5494 & 0.7397 \\
\hline 100 & 0.2479 & 0.3341 & 0.4474 & 0.4851 & 0.4567 & 0.5539 & 0.5999 & 0.5013 \\
\hline
\end{tabular}

b) For estimating the efficiency, as in section 2.2 Case II also 80 random samples are simulated from binomial distributions, $X: B(n, \theta)$. For each $\theta=0.2: 0.1: 0.9$ and for each $n=10: 10: 100$. In each case the efficiency ef $f$ and ef $f^{*}$ of each simulated sample is computed in Tables 3 and 4 respectively from the Equations (2.6) and (2.7). 
Table 3: Estimated efficiency of sample of size $n$ from binomial distribution with parameter $\theta$ by Shannon entropy

\begin{tabular}{|c|c|c|c|c|c|c|c|c|}
\hline$n$ & 0.2 & 0.3 & 0.4 & 0.5 & 0.6 & 0.7 & 0.8 & 0.9 \\
\hline 10 & 0.4073 & 0.4733 & 0.5597 & 0.6521 & 0.6077 & 0.6834 & 0.7785 & 0.6945 \\
\hline 20 & 0.6092 & 0.6946 & 0.6746 & 0.7408 & 0.8231 & 0.7698 & 0.7483 & 0.8421 \\
\hline 30 & 0.5960 & 0.6613 & 0.7339 & 0.7334 & 0.8026 & 0.8982 & 0.9599 & 0.7911 \\
\hline 40 & 0.6456 & 0.7057 & 0.7633 & 0.8217 & 0.8554 & 0.9848 & 0.9553 & 0.8220 \\
\hline 50 & 0.6515 & 0.6708 & 0.7771 & 0.8301 & 0.9836 & 0.9139 & 0.9653 & 0.9551 \\
\hline 60 & 0.6544 & 0.6847 & 0.7311 & 0.8446 & 0.9026 & 0.9199 & 0.9953 & 0.8018 \\
\hline 70 & 0.6505 & 0.6938 & 0.7715 & 0.9062 & 0.9474 & 0.9560 & 0.9534 & 0.9297 \\
\hline 80 & 0.6752 & 0.7181 & 0.7680 & 0.8155 & 0.9041 & 0.9902 & 0.9927 & 0.9815 \\
\hline 90 & 0.6603 & 0.7264 & 0.7922 & 0.8540 & 0.9330 & 0.9919 & 0.9907 & 0.9370 \\
\hline 100 & 0.6595 & 0.7331 & 0.7832 & 0.8839 & 0.9230 & 0.9911 & 0.9904 & 0.9084 \\
\hline
\end{tabular}

Table 4: Estimated efficiency of sample of size $\mathrm{n}$ from binomial distribution with parameter $\theta$ by A-entropy

\begin{tabular}{|c|c|c|c|c|c|c|c|c|}
\hline$n$ & 0.2 & 0.3 & 0.4 & 0.5 & 0.6 & 0.7 & 0.8 & 0.9 \\
\hline 10 & 0.1979 & 0.1951 & 0.1000 & 0.1077 & 0.2978 & 0.7094 & 0.9830 & 0.1569 \\
\hline 20 & 0.3519 & 0.3135 & 0.2282 & 0.2200 & 0.3508 & 0.9646 & 0.6733 & 0.1395 \\
\hline 30 & 0.3321 & 0.1488 & 0.1361 & 0.3019 & 0.2660 & 0.6755 & 0.7425 & 0.1740 \\
\hline 40 & 0.4159 & 0.4870 & 0.3313 & 0.2406 & 0.3608 & 0.7391 & 0.5980 & 0.1499 \\
\hline 50 & 0.4028 & 0.4256 & 0.2986 & 0.2583 & 0.3702 & 0.9342 & 0.9365 & 0.1403 \\
\hline 60 & 0.3255 & 0.2642 & 0.2170 & 0.2463 & 0.3043 & 0.6762 & 0.7232 & 0.1953 \\
\hline 70 & 0.3125 & 0.3641 & 0.2605 & 0.2342 & 0.2705 & 0.8284 & 0.9497 & 0.1739 \\
\hline 80 & 0.3455 & 0.3803 & 0.2564 & 0.2607 & 0.3565 & 0.6122 & 0.9991 & 0.1804 \\
\hline 90 & 0.2396 & 0.3047 & 0.2214 & 0.2532 & 0.2610 & 0.7851 & 0.9938 & 0.1743 \\
\hline 100 & 0.3498 & 0.3603 & 0.2600 & 0.3097 & 0.3505 & 0.6255 & 0.7963 & 0.1493 \\
\hline
\end{tabular}

c) The Poisson distribution $X: P(\theta)$ is used for simulated 80 random samples as in section 2.3 Case III, for each $\theta=6: 1: 13$ and for each $n=10: 10: 100$. In each case the efficiency eff and ef $f^{*}$ of each simulated sample is computed in Tables 5 and 6 respectively from the Equations (2.8) and (2.9). Table 5. Estimated efficiency of sample of size $\mathrm{n}$ from Poisson distribution with parameter ? by Shannon entropy. 
Table 5: Estimated efficiency of sample of size $n$ from Poisson distribution with parameter $\theta$ by Shannon entropy

\begin{tabular}{|c|c|c|c|c|c|c|c|c|}
\hline$n$ & 6 & 7 & 8 & 9 & 10 & 11 & 12 & 13 \\
\hline 10 & 0.6949 & 0.6950 & 0.5700 & 0.7672 & 0.6225 & 0.6424 & 0.7672 & 0.7673 \\
\hline 20 & 0.8425 & 0.7375 & 0.8262 & 0.7887 & 0.8674 & 0.7900 & 0.8266 & 0.8787 \\
\hline 30 & 0.8103 & 0.7787 & 0.8321 & 0.8410 & 0.8685 & 0.9196 & 0.8794 & 0.7862 \\
\hline 40 & 0.7715 & 0.8552 & 0.8046 & 0.8222 & 0.8870 & 0.9101 & 0.8425 & 0.9410 \\
\hline 50 & 0.8721 & 0.8649 & 0.8535 & 0.8964 & 0.8553 & 0.8731 & 0.9382 & 0.9743 \\
\hline 60 & 0.8066 & 0.9135 & 0.8781 & 0.8854 & 0.9652 & 0.9676 & 0.9306 & 0.9664 \\
\hline 70 & 0.7718 & 0.8562 & 0.9251 & 0.9256 & 0.9448 & 0.9877 & 0.9284 & 0.9465 \\
\hline 80 & 0.8205 & 0.8599 & 0.8545 & 0.8963 & 0.9327 & 0.9637 & 0.9305 & 0.9824 \\
\hline 90 & 0.8401 & 0.8864 & 0.8743 & 0.9323 & 0.9601 & 0.9474 & 0.9066 & 0.9590 \\
\hline 100 & 0.8033 & 0.9360 & 0.9336 & 0.8946 & 0.9169 & 0.9428 & 0.9841 & 0.9989 \\
\hline
\end{tabular}

Table 6: Estimated efficiency of sample of size $n$ from Poisson distribution with parameter $\theta$ by A-entropy

\begin{tabular}{|c|c|c|c|c|c|c|c|c|}
\hline$n$ & 6 & 7 & 8 & 9 & 10 & 11 & 12 & 13 \\
\hline 10 & 0.4530 & 0.7562 & 0.6845 & 0.7855 & 0.4465 & 0.6046 & 0.3455 & 0.5623 \\
\hline 20 & 0.6928 & 0.9027 & 0.8004 & 0.8857 & 0.7098 & 0.5003 & 0.7089 & 0.5867 \\
\hline 30 & 0.9992 & 0.9294 & 0.5332 & 0.9940 & 0.7733 & 0.5780 & 0.8140 & 0.6902 \\
\hline 40 & 0.5653 & 0.7833 & 0.5771 & 0.9759 & 0.7160 & 0.9094 & 0.8569 & 0.9113 \\
\hline 50 & 0.6678 & 0.6197 & 0.9025 & 0.8160 & 0.9757 & 0.7775 & 0.9007 & 0.6946 \\
\hline 60 & 0.6239 & 0.6793 & 0.6916 & 0.8603 & 0.9464 & 0.8957 & 0.8362 & 0.8231 \\
\hline 70 & 0.7765 & 0.6358 & 0.7023 & 0.7731 & 0.9113 & 0.7219 & 0.7805 & 0.9144 \\
\hline 80 & 0.6529 & 0.5911 & 0.7682 & 0.7720 & 0.8446 & 0.6312 & 0.6041 & 0.8502 \\
\hline 90 & 0.4900 & 0.8213 & 0.8412 & 0.8171 & 0.7598 & 0.7442 & 0.7130 & 0.7734 \\
\hline 100 & 0.7157 & 0.9224 & 0.7485 & 0.8633 & 0.7386 & 0.7219 & 0.7201 & 0.6014 \\
\hline
\end{tabular}

\section{CONCLUSIONS}

The calculations of the estimated relative efficiency of entropies given in [4], [15] are derived for the cases where the distribution of sample size that selected are geometric, 
binomial and Poisson. These calculations that given by using Tables 1 to 6 in example 1 shows that:

(a) $0 \leq$ eff $\leq 1$ and $0 \leq e f f^{*} \leq 1$.

(b) The estimated relative efficiency increases with sample size $\mathrm{n}$ in both Shannon and Awad sup-entropies.

(c) The estimated relative efficiency which is calculated from Shannon and Awad supentropies are closed to each other especially for Poisson distribution.

More precisely one may take the sample size $\mathrm{n}$ as follow:

1. (1) For the case of geometric distribution, calculations shows that when size of the sample $n=50$, for p.d. $f=f(x, \theta), \theta=0.5$ and hence ef $f \approx 94$ by Table 1 .

2. For the binomial distributions case, we considered the size $n=80$ and $\theta=0.7$, then ef $f \approx 99$ by Table 3 .

3. For the case of Poisson distribution, $n=40$ and $\theta=9$ and hence ef $f^{*} \approx 99$ by Table 6.

\section{ACKNOWLEDGMENTS}

The author would like to thank Professor I.H.Elsanousi for his comments,suggestions and recommendations.

\section{REFERENCES}

[1] C.J. Adcock,1997, The Choice of sample size and the maximum expected utilityComments on the paper by Lindley. The Statistician Vol.46 (2):155-162.

[2] C.J. Adcock, 1997,Sample size determination: A Review. The Statistician Vol.46 (2): 261-283.

[3] A.M.Awad,1996. Entropy of a partition and efficiency of a sample. Abhath AlYarmouk pure sci. and eng. Vol. 5: 9- 30.

[4] A. M.Awad,1987,Statistical information measures, Dirasat (Science), XIV 12: $7-20$.

[5] A. M.Awad,and T.Al Sarie,1996. Cluster of entropy measures based on binomial distribution. Abhath Al-Yarmouk, Vol.5: 31-69.

[6] M. M.Azzam ,And A.M.Awad,1996,Entropy measures and some distribution Approximations. Microelectron Reliab. Vol. 36(10):1569-1580.

[7] A.Feinstein,1958. Foundation of information theory. McGraw-Hill: N. Y. 
[8] M. E.Gobar,E.B.Bashier,and A.M.Awad,2014.Information measures and some distribution approximations. Journal of advances in mathematics Vol.6 (3) :10081014.

[9] M.E.Gobar,and E.B.Bashier,2014.Akaike information criterion and some distribution approximations. International J.of Math. Sci. and Engg. Appl. (IJMSEA) Vol.8 No II: 1-9.

[10] M.E.Gobar,E.B.Bashier,and A.M.Awad,2014. The best sample size in approximating some sampling distributions. International J. of Math. Sci. and Engg. Applec. (IJMSEA). Vol.8 No3: 179-192.

[11] M.E.Gobar,and E.B.Bashier,2015.Estimating sample size to approximating some sampling distributions by information measures. Journal of progressive research in mathematics Vol. 3(3):203-215.

[12] M.E.Gobar,1999. Optimal sample size in approximating some sampling Distributions. M.Sc. Thesis .University of Jordan: Jordan.

[13] L.Joseph,1997. Interval-based versus decision theoretic criteria for the choice of sample size. The Statistician Vol.46 (2): 145-149.

[14] D.V.Lindley,1997. The Choice of sample size. The Statistician Vol.46 (2): 129138.

[15] E.E.Shannon,1948.A mathematical theory of communication. Bulletin of systems Technology J. 27: 379-423 and 623-659.

[16] S.A.Singh, and Masuku, B.M. 2014. Sampling techniques and determination of sample size in applied statistics research. An overview. Introduction journal of Economics. commerce and management Vol.11(11): 1-22. 